\title{
Bayesian Analysis of Two-Parameter Exponentiated Log-logistic Distribution
}

\author{
Arun Kumar Chaudhary, Ph.D. \\ Associate Professor, Nepal Commerce Campus, T.U.
}

\begin{abstract}
In this paper, the parameters of the two-parameter exponentiated log-logistic distribution based on a complete sample are estimated using the Markov chain Monte Carlo (MCMC) method. In order to perform full Bayesian analysis of the two-parameter exponentiated log-logistic distribution, the procedures are developed using the MCMC simulation method in OpenBUGS, established software. The researcher has obtained the Bayes estimates of the parameters and their probability intervals are presented. The researcher has also discussed the estimation of the reliability function. For illustration under independent gamma priors, the real data set is considered.
\end{abstract}

Keywords - Bayesian estimation, Two-parameter exponentiated Log-logistic distribution, Markov chain Monte Carlo, Gamma Prior.

\section{Introduction}

The log-logistic distribution is very useful in survival analysis since it has a nonmonotonic hazard function, (Bennett, 1983) and (Tadikamalla and Johnson, 1982). When dealing with censored data, which makes this distribution more convenient is that the shape of this distribution has a more manageable form than that of the log-normal, even though the shape is similar. (Srivastava and Shukla, 2008) studied the log-logistic distribution as a step-stress model. (Balakrishnan and Malik, 1987) gave the moments of order statistics from the truncated log-logistic distribution. This distribution has been also studied by (Howlader and Weiss, 1992). (Lawless, 2003), (Lee and Wang, 2003) and (Murthy et al., 2004) provide an excellent review for the log-logistic distribution.

Based on modifications such as adding parameters to the existing models, new classes of models have been proffered in recent years. Adding one or more parameters to distribution makes it richer and more flexible for modeling data.

There are different ways for adding a parameter(s) to a distribution. (Marshall and Olkin (1997, 2007)) added one positive parameter to a given (general) survival function. As described by (Marshall and Olkin, 2007) and (Klugman et al., 2012), an exponentiated distribution can be easily constructed. It is based on the observation that by raising any baseline Cumulative Distribution Function (CDF) $F_{\text {baseline }}(x)$ to arbitrary power $\alpha>0$, a new $\mathrm{CDF}$

$$
F(x)=\left(F_{\text {baseline }}(x)\right)^{\alpha} ; \alpha>0
$$

is obtained with the additional parameter $\alpha$.

This idea led several authors to consider extensions from usual survival distributions. The exponentiated Weibull distribution was considered by (Mudholkar and Srivastava, 1993) as a generalization of the Weibull distribution. The reliability test plan for exponentiated log-logistic distribution was studied by (Rosaiah, et al. $(2006,2007)$ ). The Kumaraswamy-log-logistic distribution was introduced by (Santana 
et al., 2012) which includes exponentiated log-logistic distribution. Chaudhary and Kumar (2014) obtained the maximum likelihood and Bayes estimators for the parameters, the posterior predictive check procedure for evaluating the model fit and the reliability function of the three-parameter exponentiated log-logistic distribution using Markov Chain Monte Carlo simulation method.

The CDF of the log-logistic distribution is given by

$$
F_{L L}(x ; \beta, \lambda)=\frac{(x / \lambda)^{\beta}}{1+(x / \lambda)^{\beta}} ;(\beta, \lambda)>0, \quad x>0
$$

where $\beta>0$ is the shape and $\lambda>0$ is the scale parameter.

The CDF of the exponentiated log-logistic(ELL) distribution is defined by raising $F_{L L}(x)$ to the power of $\alpha$, namely $F(x)=\left(F_{L L}(x)\right)^{\alpha}$. The distribution function of ELL distribution with three parameters is given by

$$
F(x ; \alpha, \beta, \lambda)=\left\{\frac{(x / \lambda)^{\beta}}{1+(x / \lambda)^{\beta}}\right\}^{\alpha} ;(\alpha, \beta, \lambda)>0, \quad x>0
$$

Where shape parameters are $\alpha>0$ and $\beta>0$ and the scale parameter is $\lambda>0$. The model reduces to the $\log$-logistic distribution when $\alpha=1 . \lambda=1$ can be assumed without any loss of generality since $\lambda$ it is the scale parameter. When $\lambda=1$ three-parameter ELL distribution reduces to twoparameter ELL distribution and we shall denote it as $\operatorname{ELL}(\alpha, \beta)$.

The rest of the article is arranged as given. The model and its features are introduced in Section 2. In Section 3, the researcher has discussed the Bayesian model formulation including the priors, posterior, Gibbs sampler and its implementation in OpenBUGS. The real data set is considered for Bayesian analysis in Section 4. The full Bayesian analysis under independent gamma of priors for the data set using the Markov chain Monte Carlo (MCMC) simulation method in OpenBUGS, established software, is presented in Section 5. The Bayes estimates of the parameters and their probability intervals based on posterior samples are presented. The posterior analysis is performed and we have also estimated the reliability function. Conclusions are given in Section 6.

\section{The Two -Parameter Exponentiated Log-logistic Model}

The Cumulative Distribution Function (CDF) of two-parameter exponentiated log-logistic (ELL) distribution is given by

$$
F(x ; \alpha, \beta)=\left\{\frac{x^{\beta}}{1+x^{\beta}}\right\}^{\alpha} ;(\alpha, \beta)>0, \quad x>0
$$

where the shape parameters are $\alpha>0$ and $\beta>0$.

And the corresponding Probability Density Function (PDF) with shape parameters $\alpha>0$ and $\beta>0$ is given by

$$
f(x ; \alpha, \beta)=\frac{\alpha \beta x^{\alpha \beta}}{x\left[1+x^{\beta}\right]^{\alpha+1}} \quad ;(\alpha, \beta)>0, x>0 .
$$

The reliability/survival function is given by

$$
R(x ; \alpha, \beta)=1-\left\{\frac{x^{\beta}}{1+x^{\beta}}\right\}^{\alpha} \quad ;(\alpha, \beta)>0, \quad x>0 .
$$

The hazard rate function with shape parameters $\alpha>0$ and $\beta>0$ is 


$$
h(x ; \alpha, \beta)=\frac{\alpha \beta x^{\alpha \beta}}{x\left(1+x^{\beta}\right)^{\alpha+1}}\left(1-\left\{\frac{x^{\beta}}{1+x^{\beta}}\right\}^{\alpha}\right)^{-1} ;(\alpha, \beta)>0, x>0 .
$$

The quantile function is given by

$$
x_{p}=\left(p^{-1 / \alpha}-1\right)^{-1 / \beta} ; 0<p<1 .
$$

The random deviate can be generated from $\operatorname{ELL}(\alpha, \beta)$ by

$$
x=\left(u^{-1 / \alpha}-1\right)^{-1 / \beta} ; 0<u<1
$$

where $u$ have the $U(0,1)$ distribution.
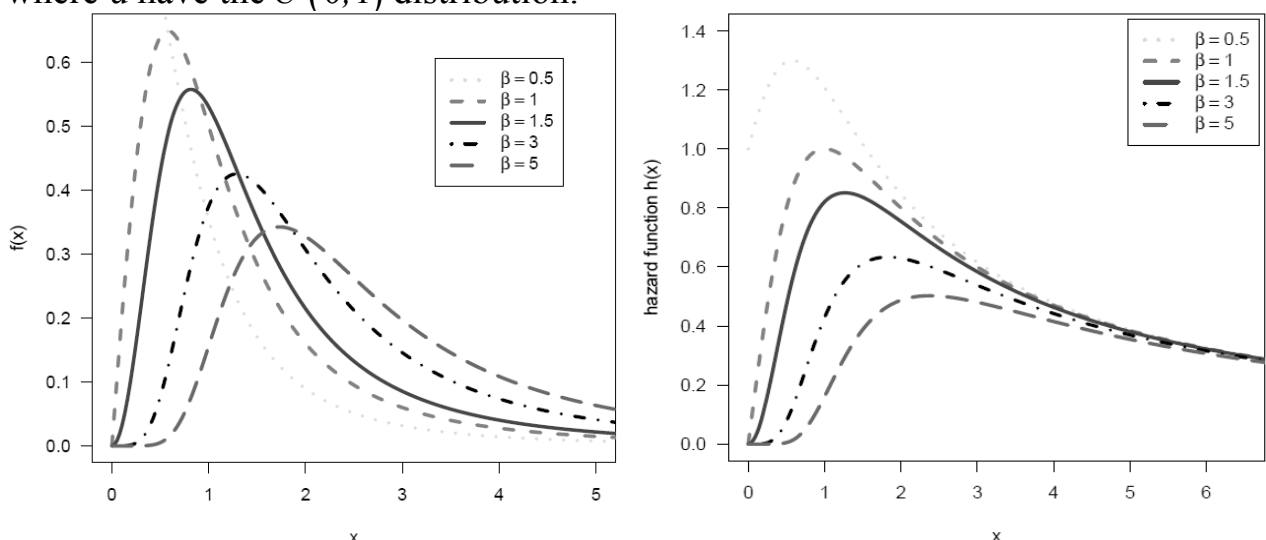

Figure 1: The probability density function (Left panel); The hazard function (Right panel) of ELL( $\alpha, \beta)$ distribution for $\alpha=1$ and different values of $\beta$.

Some of the typical $\operatorname{ELL}(\alpha, \beta)$ density functions for different values of $\beta$ and $\alpha=$ lare depicted in Figure 1 (left panel). It is evident from Figure 1 that the density function of the ELL distribution can take different shapes. Figure 1(right panel) exhibits the different hazard rate functions of $E L L(\alpha, \beta)$ distribution.

\section{Bayesian model formulation}

In this section, the Bayes estimates of the shape parameters assuming independent gamma priors for both the parameters $\alpha \beta$ and are provided. For $\operatorname{ELL}(\alpha, \beta)$ the Bayesian model is constructed by specifying a prior distribution for $\alpha \beta$ and, and then multiplying with the likelihood function to obtain the posterior distribution function. Given a set of data $\mathrm{x}=(\mathrm{x} 1, \mathrm{x} 2 \ldots \mathrm{xn})$, the likelihood function is

$$
L(\alpha, \beta \mid \underline{x})=\alpha^{n} \beta^{n} \prod_{i=1}^{n} \frac{x_{i}^{\alpha \beta-1}}{\left(1+x_{i}^{\beta}\right)^{\alpha-1}} .
$$

\section{Prior distributions}

Denote the prior distribution $\alpha \beta p(\alpha, \beta)$ as. The joint posterior is $p(\alpha, \beta \mid \underline{x}) \quad \propto \quad L(\alpha, \beta \mid \underline{x}) p(\alpha, \beta)$.

We assume the independent gamma priors for $\alpha \sim G(a, b)$ and $\beta \sim G(c, d)$ as 


$$
p(\alpha)=\frac{b^{a}}{\Gamma(a)} \alpha^{a-1} \mathrm{e}^{-b \alpha} ; \alpha>0,(a, b)>0
$$

and

$$
p(\beta)=\frac{d^{c}}{\Gamma(c)} \beta^{c-1} \mathrm{e}^{-d \beta} ; \beta>0,(c, d)>0 .
$$

\section{Posterior distribution}

Combining the likelihood function with the gamma prior via Bayes' theorem gives the posterior distribution up to proportionality as

$$
\begin{aligned}
& p(\alpha, \beta \mid \underline{x}) \propto \alpha^{a+n-1} \beta^{c+n-1} \exp \{-(b \alpha+d \beta)\} T_{1} \\
& \text { where } T_{1}=\prod_{i=1}^{n} \frac{x_{i}^{\alpha \beta-1}}{\left(1+x_{i}^{\beta}\right)^{\alpha-1}} .
\end{aligned}
$$

The posterior distribution is obviously complicated and no close form inferences appear possible. Therefore, consideration of MCMC methods is proposed to simulate samples from the posterior distribution so that sample-based inferences can be easily drawn. An important Markov Chain Monte Carlo technique is the Gibbs sampler, which provides a way for extracting samples from the posterior distribution. Even though this sampling scheme was first introduced by (Geman and Geman, 1984), the demonstration of the applicability to statistical modeling for Bayesian computation was done by (Gelfand and Smith, 1990).

By sampling from each of the uni-variate full conditional distributions, in turn, a sample from a randomly complex multidimensional distribution is created by the Gibbs Sampler. That is, every time a variate value is generated from a full conditional, it is influenced by the most recent values of all other conditioning variables and, after each cycle of iteration, it is updated by sampling a new value from its full conditional. The entire generating scheme is repeated until the generating chain achieves a systematic pattern of convergence. It can be shown that after a large number of iterations the generated variates can be regarded as the random samples from the corresponding posteriors. The details of the method and the related convergence diagnostic issues have been given by (Gelman et al., 2004), (Albert, 2009), (Hamada et al., 2008), (Ntzoufras, 2009) and (Hoff, 2009). Therefore to obtain the full conditional distribution of $\alpha$ (or $\beta$ ), we need only choose the terms in the posterior, which involve parameter $\alpha \mathrm{s}$ (or $\beta$ ). The full posterior conditional distributions for $\alpha \beta$ and, are

$$
p(\alpha \mid \beta, \underline{x}) \propto \alpha^{a+n-1} \exp (-b \alpha) T_{1}
$$

$$
\begin{aligned}
& \text { and } \\
& \qquad p(\beta \mid \alpha, \underline{x}) \propto \beta^{c+n-1} \exp (-d \beta) T_{1} .
\end{aligned}
$$

As the two-parameter exponentiated log-logistic(ELL) distribution is not available in OpenBUGS., it requires incorporation of a module in ReliaBUGS, (Kumar et al., 2010) and (Lunn, 2010), a subsystem of OpenBUGS for ELL. A module dexpo.loglogistic_T(alpha, beta) is written in Component Pascal for ELL, the corresponding computer program can be obtained from authors, to perform full Bayesian analysis in OpenBUGS using the method described in (Thomas et al., 2006), (Thomas, 2010), (Kumar et al., 2010) and (Lunn et al., 2013). It is important to note that this module can be used for any set of suitable priors of the model parameters. Almost all aspects of the model in the Bayesian framework can be studied using the developed module dexpo.loglogistic_T(alpha, beta), (Kumar, 2010). 


\section{Gibbs Sampler: Implementation}

1. Select an initial value $\underline{\theta}^{(0)}=\left(\alpha^{(0)}, \beta^{(0)}\right)$ to start the chain.

2. Suppose at the ith-step, $\underline{\theta}=(\alpha, \beta)$ takes the value $\underline{\theta}^{(i)}=\left(\alpha^{(i)}, \beta^{(i)}\right)$ then from full conditionals, we generate

$$
\begin{aligned}
& \alpha^{(i+1)} \text { from } p\left(\alpha \mid \beta^{(i)}, \underline{x}\right) \\
& \beta^{(i+1)} \text { and from } p\left(\beta \mid \alpha^{(i+1)}, \underline{x}\right) .
\end{aligned}
$$

3. This completes a transition from $\underline{\theta}^{(i)} \underline{\theta}^{(i+1)}$ to.

4. Repeat Step 2, N times.

\section{MCMC output : Posterior sample}

Monitor the convergence using convergence diagnostics(trace and ergodic mean plots). Suppose that convergence has been reached after ' $\mathrm{B}$ ' iterations (the burn-in period). Discard the observations $\left(\underline{\theta}^{(1)}, \underline{\theta}^{(2)}, \ldots, \underline{\theta}^{(B)}\right)$ and retain the observations

$$
\left(\underline{\theta}^{(B+1+(j-1) L)} ; B+1+(M-1) L \leq N ; j=1,2, \ldots, M ; L \geq 1\right)
$$

which are viewed as an independent sample from the stationary distribution of the Markov chain which is typically the posterior distribution, where ' $\mathrm{L}$ ' is the lag (or thin interval).

Consider $\left(\underline{\theta}^{(1)}, \ldots, \underline{\theta}^{(j)}, \ldots, \underline{\theta}^{(M)}\right)$ as the MCMC output (posterior sample) for the posterior analysis

$$
\underline{\theta}^{(j)}=\left(\alpha^{(j)}, \beta^{(j)}\right) ; j=1,2, \ldots, M \text {. }
$$

Thus, after the removal of the initial iterations (produced during the burn-in period) and consideration of the appropriate lag, MCMC output is recommended as the sample. The Bayes estimates of $\underline{\theta}=(\alpha, \beta)$, under squared error loss function, using the ergodic theorem are given by

$$
\hat{\alpha}=\frac{1}{M} \sum_{j=1}^{M} \alpha^{(j)} \text { and } \hat{\beta}=\frac{1}{M} \sum_{j=1}^{M} \beta^{(j)} \text {. }
$$

An important advantage of sample-based approaches includes the routine developments for any linear and/or non-linear functions of the original parameters. It is to be noted that once the samples from the posterior are obtained, samples from the posterior of any linear and/or non-linear functions can be easily created merely by substitution. Some of such functions where reliability practitioners are often interested to include reliability, hazard rate, mean time to failure, percentiles, etc.

\section{Real Data Set}

The following real data set is considered for illustration of the proposed methodology. The data given below represent active repair times (in hours) for 46 repair times of an airborne communication transceiver. (Chhikara and Folks, 1977) fitted a two-parameter inverse Gaussian distribution. The data are presented below:

$0.2,0.3,0.5,0.5,0.5,0.5,0.6,0.6,0.7,0.7,0.7,0.8,0.8,1.0,1.0,1.0,1.0,1.1,1.3,1.5,1.5,1.5,1.5$, $2.0,2.0,2.2,2.5,2.7,3.0,3.0,3.3,3.3,4.0,4.0,4.5,4.7,5.0,5.4,5.4,7.0,7.5,8.8,9.0,10.3,22.0,24.5$ 


\section{Bayesian analysis}

The developed module dexpo.loglogistic_T(alpha, beta) in OpenBUGS is implemented for the full Bayesian analysis of the exponentiated log-logistic distribution using MCMC method. The following script can be used to get samples from the posterior arising from the model.

\section{OpenBUGS script for the Bayesian analysis of ELL distribution model}

for $(\mathrm{i}$ in $1: \mathrm{N}$ )

$\{\quad \mathrm{x}[\mathrm{i}] \sim$ dexpo.loglogistic_T(alpha, beta) \# ELL distribution

$$
\text { reliability }[\mathrm{i}]<-\mathrm{R}(\mathrm{x}[\mathrm{i}], \mathrm{x}[\mathrm{i}]) \quad \text { \# to estimate reliability }
$$

$$
\mathrm{f}[\mathrm{i}]<-\operatorname{density}(\mathrm{x}[\mathrm{i}], \mathrm{x}[\mathrm{i}]) \quad \text { \# to estimate density }
$$

\# Prior distributions of the model parameters

$$
\begin{aligned}
& \text { alpha } \sim \operatorname{dgamma}(0.001,0.001) \\
& \text { beta } \sim \operatorname{dgamma}(0.001,0.001)\}
\end{aligned}
$$

\section{Data}

$\operatorname{list}(\mathrm{N}=46, \mathrm{x}=\mathrm{c}(0.2,0.3,0.5,0.5,0.5,0.5,0.6,0.6,0.7,0.7,0.7,0.8,0.8,1.0,1.0,1.0,1.0,1.1,1.3$, $1.5,1.5,1.5,1.5,2.0,2.0,2.2,2.5,2.7,3.0,3.0,3.3,3.3,4.0,4.0,4.5,4.7,5.0,5.4,5.4,7.0,7.5,8.8,9.0$, $10.3,22.0,24.5))$

\section{Initial values}

$$
\begin{array}{ll}
\text { list }(\text { alpha } & =0.5, \text { beta }=0.5) \\
\text { list }(\text { alpha }=3.0, \text { beta }=2.5) & \text { \# Chain } 1 \\
\text { \# Chain } 2
\end{array}
$$

We run the model to generate two Markov Chains at the length of 40,000 with different starting points of the parameters. The convergence is monitored using trace and ergodic mean plots, we find that the Markov Chain converge together after approximately 2000 observations. So, burn-in of 5000 samples is more than sufficient to erase the effect of starting point (initial values). Lastly, from the posterior distribution, samples of size 7000 are created by picking up equally spaced every fifth outcome, i.e. thin=5, starting from 5001. This is done to minimize the auto correlation among the generated deviates.

Therefore, we have the posterior sample $\left(\alpha_{1}^{(j)}, \beta_{1}^{(j)}\right) ; j=1, \ldots, 7000$ from chain 1 and $\left(\alpha_{2}^{(j)}, \beta_{2}^{(j)}\right) ; j=1, \ldots, 7000$ from chain 2.

For convergence diagnostics plots, chain 1 is taken into consideration. Based on the posterior sample obtained from chain 1, only the visual summary is presented whereas, for both the chains 1 and 2 , the numerical summary is presented.

The convergence is monitored by history/ trace and ergodic means plots. The sequential plot of parameters is the plot that most often exhibits difficulties in the Markov chain. Figure 6 shows the sequential realizations of the parameters of the model. In this case, the Markov chain seems to be mixing well enough and is likely to be sampling from the stationary distribution. As the plot looks like a horizontal band, with no long upward or downward trends, we have confirmed that the chain has converged.
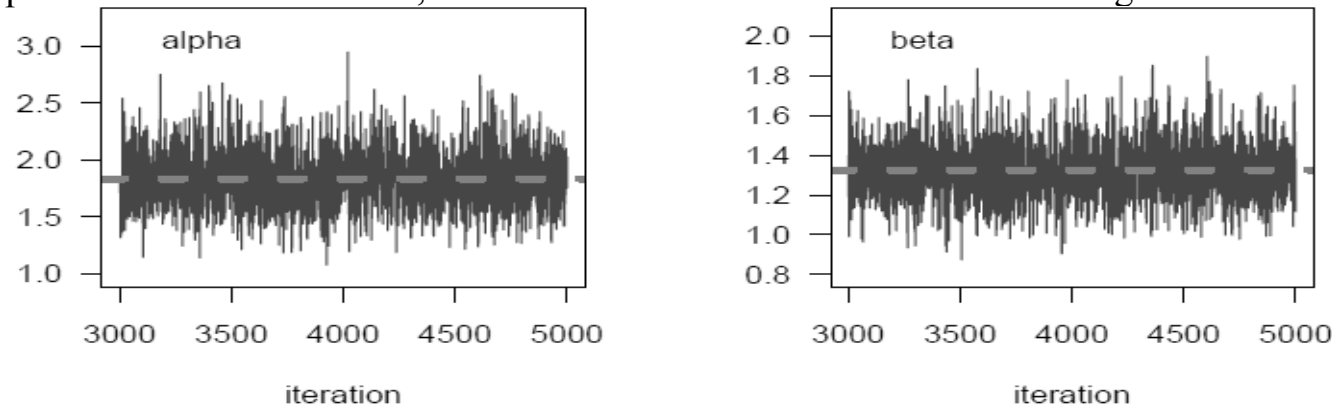

Figure 6 Trace plot of sequential realization for the parameters alpha and beta 
The running mean (ergodic mean) plot is a time series (iteration number) plot of the running mean for each parameter in the chain. The running mean is calculated as the mean of all sampled values up to and including that at a given iteration. Figure 7 shows the convergence pattern based on ergodic averages indicating the convergence of the chain.
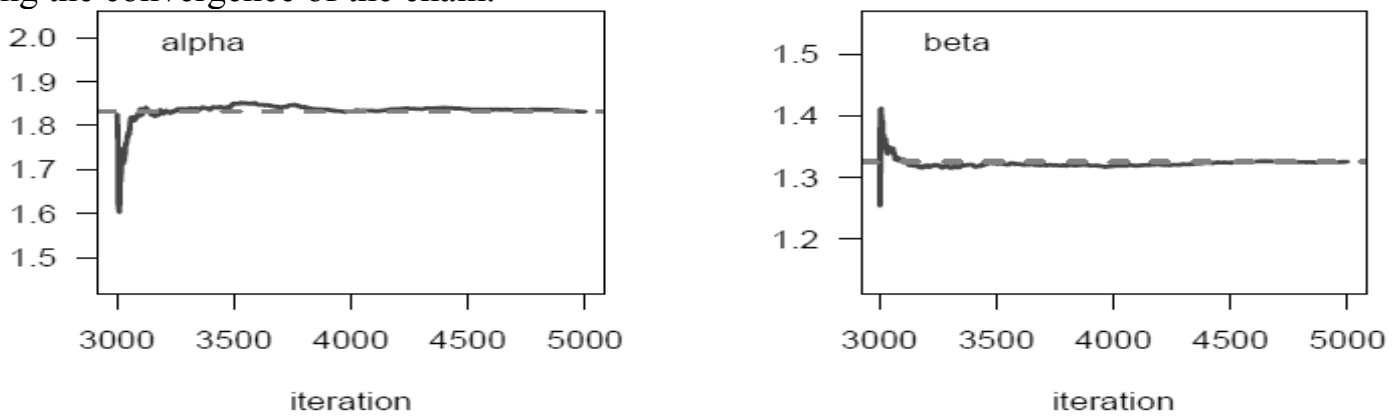

Figure 7 The Ergodic mean plots for alpha and beta

We may consider an independent sample from the target distribution i.e. posterior. Thus, we can obtain the posterior summary statistics.

\section{Posterior analysis}

\section{(a) Numerical Summary}

Thenumerical summary is presented for $\left(\alpha_{1}^{(j)}, \beta_{1}^{(j)}\right) ; j=1, \ldots, 7000$ from chain 1 and $\left(\alpha_{2}^{(j)}, \beta_{2}^{(j)}\right) ; j=1, \ldots, 7000$ from chain 2.

Various quantities of interest with their numerical values based on the MCMC sample of posterior characteristics for ELL distribution have been considered. Table 3 shows the MCMC results of the posterior mean, mode, standard deviation(SD), five-point summary statistics (minimum, first quartile, median, third quartile and maximum), 2.5th percentile, 97.5th percentile, 95\% symmetric and HPD credible intervals of the parameters $\alpha \beta$ and. In fact, we have summarized the uncertainty about the parameters. The posterior characteristics presented in Table 3 are very closed for chain 1 and chain 2 . Therefore, one can use either of the chains for posterior analysis.

The Highest probability density (HPD) intervals have computed the algorithm described by (Chen and Shao, 1999) under the assumption of the unimodal marginal posterior distribution.

Table 1

Numerical summaries based on MCMC sample of posterior characteristics for ELL distribution under gamma priors

\begin{tabular}{|l|c|c|c|c|}
\hline \multirow{2}{*}{ Characteristics } & \multicolumn{2}{|c|}{ Chain 1 } & \multicolumn{2}{c|}{ Chain 2 } \\
\cline { 2 - 5 } & alpha & beta & alpha & beta \\
\hline Mean & 1.830 & 1.327 & 1.836 & 1.328 \\
\hline Standard Deviation(S.D.) & 0.271 & 0.157 & 0.275 & 0.153 \\
\hline Monte Carlo(MC) error & 0.003 & 0.002 & 0.003 & 0.002 \\
\hline Minimum & 0.982 & 0.824 & 1.030 & 0.791 \\
\hline First Quartile $\left(\mathrm{Q}_{1}\right)$ & 1.645 & 1.218 & 1.640 & 1.222 \\
\hline Median & 1.817 & 1.322 & 1.826 & 1.320 \\
\hline Third Quartile $\left(\mathrm{Q}_{3}\right)$ & 2.001 & 1.430 & 2.012 & 1.427 \\
\hline Maximum & 3.179 & 2.062 & 2.904 & 2.200 \\
\hline Mode & 1.801 & 1.323 & 1.857 & 1.303 \\
\hline 2.5th Percentile $\left(\mathrm{P}_{25}\right)$ & 1.336 & 1.040 & 1.344 & 1.048 \\
\hline 97.5th Percentile( $\left.\mathrm{P}_{97.5}\right)$ & 2.400 & 1.654 & 2.412 & 1.632 \\
\hline 95\% Credible Interval & $(1.336,2.400)$ & $(1.040,1.654)$ & $(1.344,2.412)$ & $(1.048,1.642)$ \\
\hline 95\% HPD Credible Interval & $(1.318,2.374)$ & $(1.018,1.627)$ & $(1.329,2.393)$ & $(1.046,1.640)$ \\
\hline
\end{tabular}




\section{(b) Visual summary: Histogram and Kernel density estimates}

Figure 8 and Figure 9 represent the histogram and marginal posterior density for $\alpha$ (left panel) and for $\beta$ (right panel).

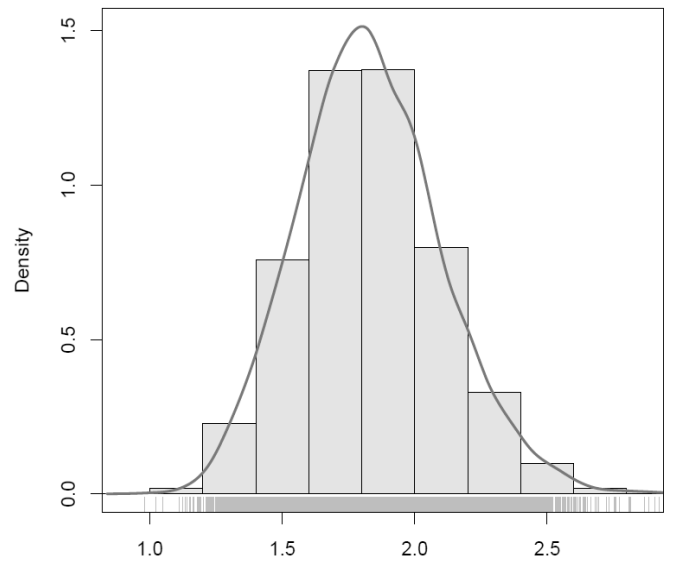

Figure 8 Histogram and marginal posterior density of $\alpha$.

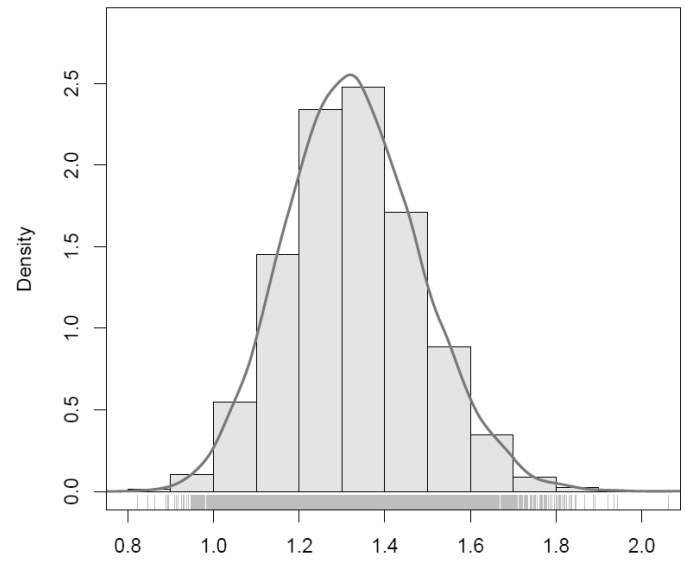

Figure 9 Histogram and marginal posterior density of $\beta$.

We have also plotted the actual realizations of parameter values along the $\mathrm{x}$-axis, which is known as a "rug" plot. Insights on skewness, behavior in the tails, presence of multi-modal behavior, and data outliers can be provided by histograms; histograms can be compared to the fundamental shapes associated with standard analytic distributions. The kernel density estimates have been plotted using $\mathrm{R}$ software with the assumption of Gaussian kernel and properly chosen values of the bandwidths. It can be seen that $\alpha$ and $\beta$ both are slightly positively skewed.

\section{Comparison with MLE}

The researcher has used a graphical method for the comparison of Bayes estimates with ML estimates. In Figure 10, the density functions $f(x ; \hat{\alpha}, \hat{\beta})$ using MLEs and Bayesian estimates, computed via MCMC samples under gamma priors, are plotted. It is clear from Figure 10 that the MLEs and the Bayes estimates with respect to the gamma priors are quite close and fit the data very well.

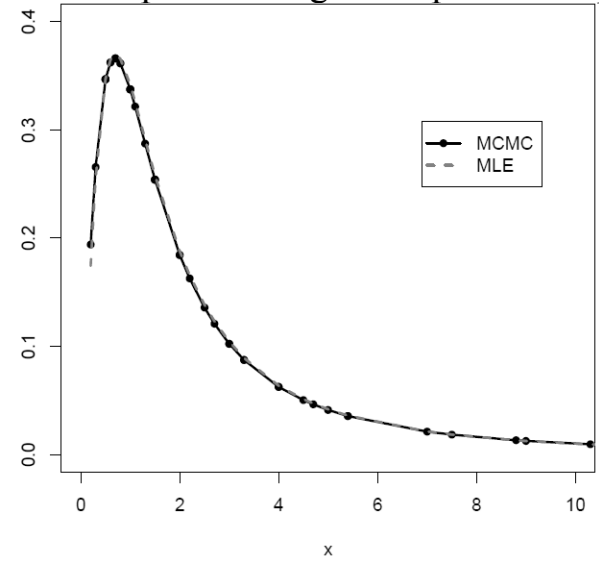

Figure 10 The density functions using $M L$ and Bayesian estimates

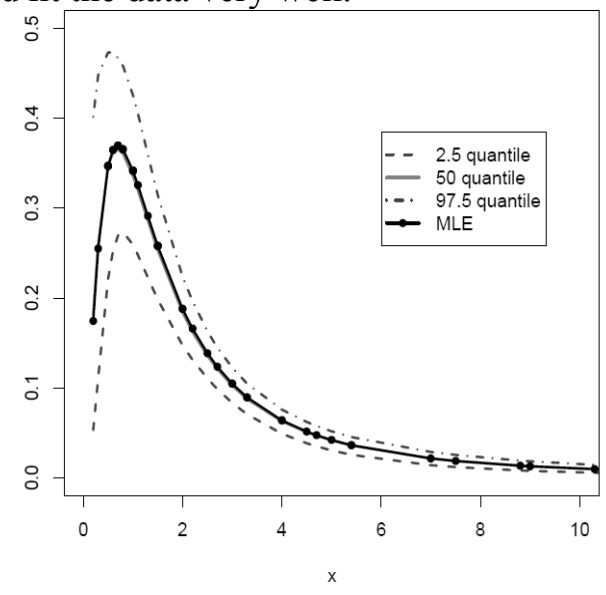

Figure 11 Density estimates

Further support for this finding can be obtained by inspecting Figure 11. In Figure 15 we have plotted $2.5^{\text {th }}, 50^{\text {th }}$ and $97.5^{\text {th }}$ quantiles of the estimated density based on the MCMC sample $\left(\alpha_{1}^{(j)}, \beta_{1}^{(j)}\right) ; j=1, \ldots, 7000$ . Here the density is computed at each data point for 7000 posterior samples. The density corresponding to MLE has been plotted using the "plug-in" estimates of the parameters. It shows that we have a fairly good model for the given data set. 


\section{Estimation of the reliability function}

In this section, our main aim is to demonstrate the effectiveness of the proposed methodology. For this, we have estimated the reliability function using MCMC samples under gamma priors. Since we have an effective MCMC technique, we can estimate any function of the parameters. We have used the empirical reliability function to make the comparison more meaningful. Figure 12, exhibits the estimated reliability function (dashed line: $2.5^{\text {th }}$ and $97.5^{\text {th }}$ quantiles; solid line: $50^{\text {th }}$ quantile) using Bayes estimate based on MCMC output under independent gamma priors for both the parameters and the empirical reliability function (solid line). Figure 12 shows that reliability estimate based on MCMC is very close to the empirical reliability estimates.

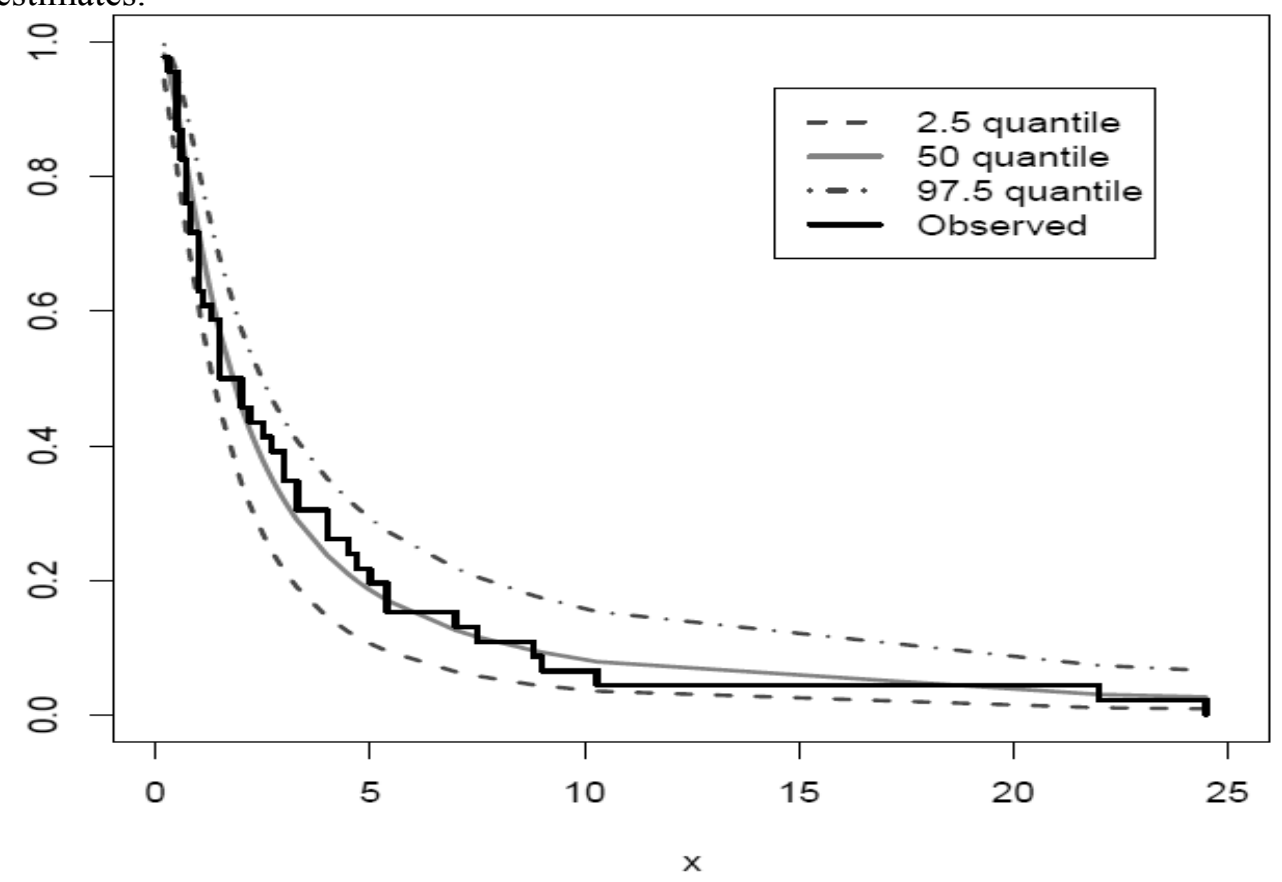

Figure 12 The empirical and estimated reliability function using $M C M C$

\section{Conclusion}

The researcher has discussed the Markov chain Monte Carlo (MCMC) method to compute the Bayesian estimates of the parameters and reliability functions of two -parameter exponentiated loglogistic distribution based on a complete sample. He has obtained the probability intervals for parameters. The MCMC method provides an alternative method for parameter estimation of the exponentiated loglogistic distribution. It is more flexible when compared with traditional methods such as the MLE method. Moreover, 'exact' probability intervals are available rather than relying on estimates of the asymptotic variances. Indeed, the MCMC sample may be used to completely summarize posterior distribution about the parameters, through kernel estimation. This is also true for any function of the parameters such as reliability and hazard functions. He has applied the developed techniques on a real data set. The paper successfully describes the scope of the Markov chain Monte Carlo (MCMC) technique in the two-parameter exponentiated log-logistic distribution. Thus, the tools developed can be applied for full Bayesian analysis of the two-parameter exponentiated log-logistic distribution

\section{References}

Albert, J. (2009). Bayesian Computation with R. 2nd edition. Springer, New York.

Balakrishnan, N. and Malik, H.J. (1987). Moments of order statistics from truncated log-logistic distribution. Journal of Statistical Planning and Inference. 17: 251-267.

Bennette, S. (1983). Log-logistic regression models for survival data. Applied Statistics. 32: 165 - 171. 
Chen, M. H., and Shao, Q. M. (1999). Monte Carlo estimation of Bayesian credible intervals and HPD intervals. Journal of Computational and Graphical Statistics. 8(1): 69-92.

Chhikara, R.S. and Folks, J.L. (1977). The inverse Gaussian distribution as a lifetime model. Technometrics. 19: 461-468.

Chaudhary, A.K.(2016). Classical estimation of two-parameter Exponentiated Log-logistic distribution. NCC Journal .1(1): PN.

Chaudhary, A.K. and Kumar, V.(2014), "Bayesian Estimation of Three- Parameter Exponentiated Loglogistic Distribution"International journal of Statistika and Mathematika,9(2),66-81.

Gelfand, A.E. and Smith, A.F.M.(1990). Sampling-based approach to calculating marginal densities. Journal of the American Statistical Association. 85: 398-409.

Gelman, A.; Carlin, J.; Stern, H., and Rubin, D. (2004). Bayesian Data Analysis. Second Edition, Chapman \& Hall, London.

German, S. and Geman, D. (1984). Stochastic Relaxation, Gibbs Distributions, and the Bayesian Restoration of Images. IEEE Transactions on Pattern Analysis and Machine Intelligence. 6: 721-741.

Gupta, R. D., and Kundu, D. (1999). Generalized exponential distributions. Australian and New Zealand Journal of Statistics. 41(2): 173 - 188.

Hamada, M.S.; Wilson, A.G.; Reese, C.S. and Martz, H.F.(2008). Bayesian Reliability. Springer, New York. Hoff, P.D. (2009). A First Course in Bayesian Statistical Methods. Springer, New York.

Howlader, H.A. and Weiss, G.(1992). Log-logistic survival estimation based on failure-censored data. Journal of Applied Statistics. 19, No. 2: 231-240.

Klugman, S.; Panjer, H. and Willmot, G. (2012) Loss Models: From Data to Decisions. (4th edition). John Wiley \& Sons, New York.

Kumar, V. (2010). Bayesian analysis of the exponential extension model. J. Nat. Acad. Math. 24: 109-128.

Kumar, V. and Ligges, U. (2011). reliaR: A package for some probability distributions. http://cran.r-project. $\mathrm{org} / \mathrm{web} /$ packages/reliaR/index.html.

Kumar, V.; Ligges, U. and Thomas, A. (2010). ReliaBUGS User Manual: A subsystem in OpenBUGS for some statistical models. version 1.0, OpenBUGS 3.2.1, http://openbugs.info/w/Downloads/

Lawless, J. F., (2003). Statistical Models and Methods for Lifetime Data. 2nd ed., John Wiley and Sons, New York.

Lee, E.T. and Wang, T.W.(2003). Statistical Methods for Survival Data Analysis. 3rd ed., John Wiley \& Sons.

Lunn, D.(2010). Recent Developments in the BUGS software. ISBA Bulletin. 17(3) :16-17.

Lunn, D.J., Andrew, A., Best, N. and Spiegelhalter, D. (2000). WinBUGS - ABayesian modeling framework: Concepts, structure, and extensibility. Statistics and Computing. 10: 325-337

Lunn, D.J.; Jackson, C.; Best, N.; Andrew, A., and Spiegelhalter, D. (2013). The BUGS Book: A Practical Introduction to Bayesian Analysis. Chapman \& Hall/CRC, London.

Marshall, A. W., and Olkin, I. (1997). A new method for adding a parameter to a family of distributions with application to the exponential and Weibull families. Biometrika. 84(3): 641-652.

Marshall, A. W., and Olkin, I. (2007). Life Distributions: Structure of Nonparametric, Semiparametric and Parametric Families. Springer, New York.

Mudholkar, G.S. and Srivastava, D.K. (1993). Exponentiated Weibull family for analyzing bathtub failurerate data. IEEE Transactions on Reliability. 42(2): 299-302.

Murthy, D.N.P., Xie, M. and Jiang, R. (2004). Weibull Models. Wiley, New York.

Nadarajah, S. and Kotz, S. (2006). The exponentiated type distributions. Acta Applicandae Mathematicae. 92: $97-111$.

Ntzoufras, I. (2009). Bayesian Modeling using WinBUGS. John Wiley \& Sons, New York

R Development Core Team (2013). R: A language and environment for statistical computing. R Foundation for Statistical Computing. Vienna, Austria. ISBN 3-900051-07-0, URL http://www.R-project.org. 
Rizzo, M. L.(2008). Statistical computing with R. Chapman \& Hall/CRC.

Rosaiah, K.; Kantam, R.R.L. and Kumar, Ch. S. (2006). Reliability test plans for exponentiated log-logistic distribution. Economic Quality Control. 21(2) : 165-175.

Rosaiah, K.; Kantam, R.R.L. and Santosh Kumar, Ch. (2007). Exponentiated log-logistic distribution - an economic reliability test plan. Pakistan Journal of Statistics. 23 (2) : $147-156$.

Santana, T.V.F.; Ortega, E.M.M.; Cordeiro, G.M. and Silva, G.O. (2012). Kumaraswamy-Log-Logistic Distribution. Journal of Statistical Theory and Applications. 11(3) : 265-291.

Srivastava, P.W. and Shukla, R. (2008). ALog-Logistic Step-Stress Model. IEEE Transactions on Reliability. 57(3): 431-434.

Tadikamalla, P. R. and Johnson, N.L. (1982). Systems of frequency curves generated by the transformation of logistic variables. Biometrika. 69: 461-465.

Thomas, A.; O'Hara, B., Ligges, U. and Sturtz, S. (2006). Making BUGS Open. R News. 6: 12-17.

Thomas, A.(2010).OpenBUGS Developer Manual. ver.3.1.2, http://www.openbugs.info/ 
